\title{
Hospital System Vulnerability to a Moderate Earthquake
}

\author{
Ammar Abdulhamid Rahal ${ }^{1}$, Ali Messabhia ${ }^{2}$, Sauce Gerard ${ }^{1}$ and Pascal Perrotin ${ }^{1}$ \\ 1. Optimization Design and Environmental Engineering Laboratory, Polytech' Savoie University, Le Bourget du Lac 73376, France \\ 2. Civil Engineering Applied Laboratory, Tebessa University, Tebessa 12002, Algeria
}

\begin{abstract}
This research aims at proposing to the technical manager of a hospital, a method for assessing the vulnerability of the hospital system in the case of a moderate earthquake. A primary case study on a hospital centre has allowed us to define the methodological basis. Also, it concludes to the need for a systematic method due to the complexity of hospital system and the specific knowledge. This "screening method" exits but it is applicable to the context of a major earthquake, where the main objective is to save life, while ours is to guarantee the continuity of hospital services. Other aims are to adapt this method in order to introduce the concept of a moderate earthquake, to take into account the environmental interactions of the hospital system and to improve the knowledge base. Subsequently, a software tool has to be developed to facilitate the implementation for the technical manager.
\end{abstract}

Key words: Vulnerability, moderate earthquake, lifelines, hospital system.

\section{Introduction}

During the earthquake of 1994 Northridge in California, several major hospitals had to be evacuated, not because of structural damages, but because of the failure of emergency generators, air regulators and the fall of ceilings fixtures. In Canada, the 1988 earthquake in Saguenay, the strongest earthquake in eastern North America recorded during the last 50 years, has caused little structural damages. The vast majority of injuries, damages and financial losses had been caused by the failure of operational and functional building components [1].

The percentage of the cost of non-structural elements at a total cost of construction is much higher in hospitals than in other buildings. Indeed, the non-structural elements represent about $60 \%$ of the value of residential housing, whereas for hospitals values range is from $85 \%$ to $90 \%$, mainly due to the cost of medical equipment [2].

The seismic risk management requires a multidisciplinary process [3], in particular engineering, and it exhibits a multi-steps cycle in which the

Corresponding author: Ali Messabhia, Dr., research director, research fields: civil engineering and conception. E-mail: messabhia@yahoo.fr. vulnerability assessment is a very important one [4].

The overall objective is to find relevant information to assess hospital performances in seismic zones in order to detect the actions needed to reduce the vulnerability for a moderate earthquake. While in countries where seismicity is low or moderate and the collapse of hospitals has not been occurred, these establishments had suffered significant non-structural damages that have put hospitals out of service.

Our research is directed towards understanding the hospital system, including hospital services, in order to enable the hospital technical manager to better prevent potential functional danger in case of a moderate earthquake. Based on a case study, realized on a hospital center, we define the methodological requirements which are influenced by two key parameters: the cost and the competence.

This document successively presents the methodology for the case study and the main conclusions, sums up the characteristics of an existing method which is very close to our problematic, and exposes the evolution of this one to adapt it to the vulnerability assessment of the hospital system in the case of a moderate earthquake. 


\section{State of the Art}

Most existing methods and approaches of vulnerability assessment focus on the structural part are listed as below:

(1) Probabilistic approach, using damage probabilistic matrix [5];

(2) Deterministic approach, using vulnerability or fragility curves [6];

(3) Classification of structure vulnerability, using vulnerability indices, the most representative [7]: wind cross $(C v)$, quality of materials $(M q)$, irregularity in elevation (Ie), irregularity in plane $(I P)$, quality of execution $(E q)$, intervention on buildings $(I s)$, short column (Cs), soil conditions $(\mathrm{Sc})$, etc.

The vulnerability index (V) for each structure is equal to the sum of products at all levels of assessment of vulnerabilities on their weights.

In return, the vulnerability of non-structural elements or functional ones is briefly developed, aside fault, causes and events trees.

\section{Notion of Moderate Earthquake}

The structures are designed and built against the major earthquake (statutory), but through the whole structure's life, this major earthquake has a little probability to occur. On the other hand, a moderate earthquake has a high probability to occur and even several times during the building's life. In this context, we can distinguish a moderate earthquake of the major one in relation to the responsibility of the state and the technical manager:

(1) State's responsibility: Usually, the state takes care of major natural disasters (against property) or humanities (against persons); Otherwise, it is responsible of large scale disasters that exceed the capacity of the individuals or society (such as floods, fires, earthquakes and so on);

(2) Technical hospital manager's responsibility: The technical hospital manger is a person who is liable when the earthquake does not reach the regulatory level and it puts down necessary services provided by his establishment. Effectively, the hospital manager is responsible for providing services, at least, of which critical ones after a non-major earthquake.

\section{Vulnerability Assessment Process}

The approach, which has been used for hospital vulnerability assessment and developed in our case study, is organized as follows (Fig. 1).

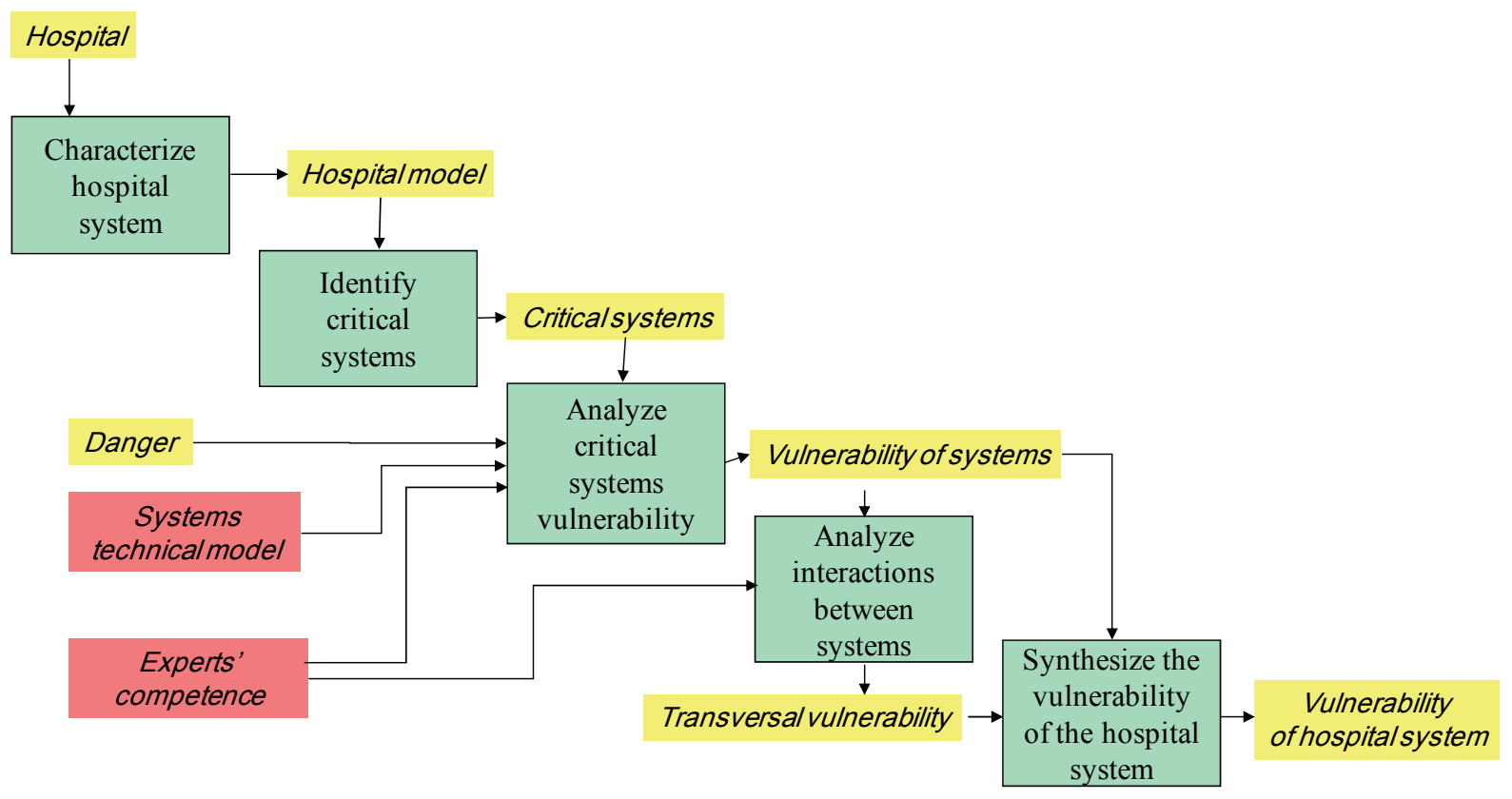

Fig. 1 Process of hospital system vulnerability analysis. 


\subsection{Characteristics of Hospital System}

A hospital is a very particular building in its structure, as well as in its organization involving a very complex industrial process. This stage aims at defining to an exhaustive way of the hospital's organization, i.e., the medical hospital corps and all attached services [8].

Generally, components constituting a hospital are classified as follows:

- structural system (frames, beams, colons, floors, etc.);

- functional hospital system (patients' flow, units' care, sterilization's ones, etc.);

- technical hospital system (lighting, heating, air conditioning, elevators, etc.);

- biomedical system (diagnostic equipment's, therapeutic equipment's, etc.).

\subsection{Identification on Critical Systems}

After the earthquake, the technical hospital manager is responsible for ensuring the continuity of services, and then there is the question: How much hospital systems required for emergency services are critical? Several visits to the studied hospital center, in cooperation with its technical staff have allowed us to characterize these critical services, i.e., the lifelines (such as water, electricity, oxygen and void, etc.).

\subsection{Analysis on Critical Systems Vulnerability}

The analysis at a primary level is based on visual

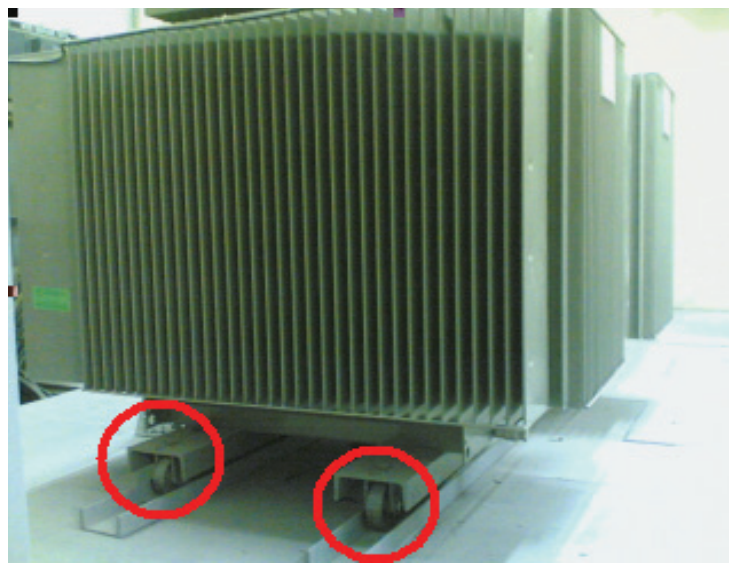

remarks, either favorable or unfavorable, in relation to the seismic response of each component of every critical system. This analysis is similar to "walk-down" one [9].

Eventually, scoring the vulnerability of a system essentially depends on our skill.

4.3.1 Analysis on Interactions between Systems (Transversal Vulnerability)

Considering that networks and pipelines are often grouped in cages or under the ceiling, falling or damaged ones can be affected by the other networks. These interactions are, first of all, analyzed in a visual way. Secondly, the interactions predictable dangerous are subjected to a deeper analysis.

4.3.2 Synthesis on the Vulnerability of the Hospital System

The judgment of the vulnerability of vital networks and the consequent vulnerability of the hospital system are done by the analyzer, based on favorable points compared to those unfavorable ones. The judgment remains, at the limit, subjective, meaning it may be different from an analyzer to another one.

\section{Vulnerability Analyses-Case Study}

\subsection{Results of Proposed Approach}

At the end of this diagnostic vulnerability analysis, we experts find that constructive dispositions against the effects of moderate earthquake are incoherent, insufficient and inadequate, as shown in Fig. 2. Without

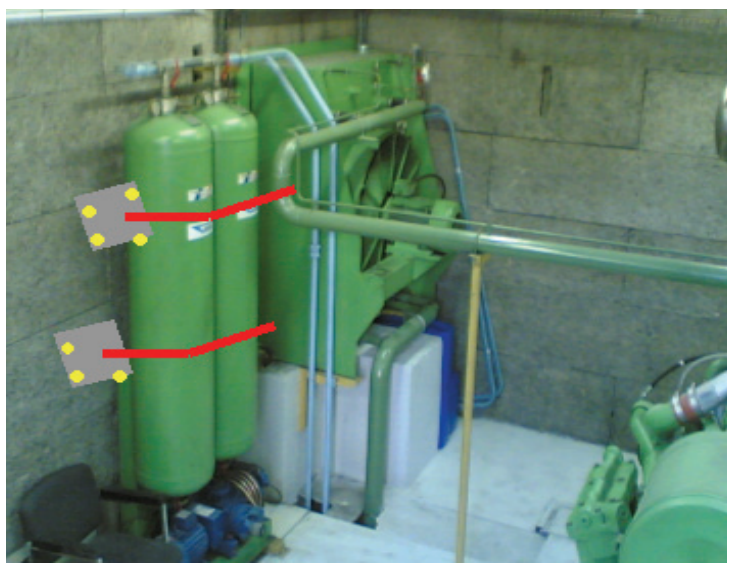

Fig. 2 Transformer on rolling support and bottles of air without strap. 
describing the result of this experiment in detail, we can resume the main conclusion as following:

- This judgment is very subjective: Two experts could produce quite different results;

- This diagnosis could be very long without a specific organization;

- It requires expert knowledge, usually not available in the technical services of the hospital;

- More detailed the results are, more complex the analysis is;

- To have recourse to a specialist (office), the cost is expensive.

Advantageously, this methodological basis is already existent previously as "screening method" [10].

\subsection{Screening Method's Description}

This is a method developed by the MCEER (Multidisciplinary Center for Earthquake Engineering Research) during a program to reduce earthquake losses [11] and defined as below.

"This method recapitulates several years of research to develop a detailed methodology to evaluate and improve the reliability of systems equipment functional after the earthquake. It is envisaged that this method could be used by engineers, asset manager, owners, technicians and others interested in evaluating and improving the functional capacity of systems and equipment. The approach is quite simple as it is described below:

- Systems definition: It requires input from appropriate facility operators and engineers to identify which systems are required for life-safety purposes and which systems are required for normal operations. For each system, it can graphically sketch system process in a logic diagram, identifying critical components, system dependencies, and redundancies;

- Evaluation of individual components: It performs a rapid visual screening inspection of each of system components;

- Systems evaluations: It develops the scores for each subsystem and system in conjunction with the logic diagram, using the scores for the individual components. The logic diagrams will help to identify weak links in the system.

The scoring methodology for an individual component uses the following logic:

- Each component is assigned a basic score that is a function of the performance history of that type of equipment and the seismicity of the site;

- The basic scores are modified by PMFs (performance modification factors) which indicate the decrease of reliability due to specific configurations or details that may be presented in an equipment installation;

- The evaluation and checklist are completed since the basic score and all applicable PMFs are identified;

- The equipment item is assigned a score which is equal to its basic negative maximum (worst case) in applicable PMF.”

\subsection{Advantages and Drawbacks of Screening Method}

Screening method allows a rapid assessment of the seismic reliability, neither very strict nor very detailed, which is intended to industrial and nuclear fields. However, it can be extended to urban areas (buildings) because some types of buildings are found in equipment and systems (networks) which are more or less similar to those in factories. For example, hospitals include systems which are quite complex, biomedical (network oxygen, avoid, etc.) or technical (water, electricity, air conditioning, etc.).

\subsubsection{Seismicity of the Site}

In the method, the seismicity of the site is divided into four zones, corresponding to several intensities of a major earthquake.

\subsubsection{Localization of the Component}

The method divides the building into three thirds (lower, middle and upper) according to the height of the building (the number of floors).

5.3.3 Dependency of Performance Modification Factors

PMFs are considered as independents, but it seems 
that this is not necessary the case. For example, if the anchor is defined as inadequate and the component is timeworn, it is obvious that the damages are augmentatives.

\section{Evolution of the Method}

We propose to adapt this screening method to our problematic into the following two directions.

\subsection{The Knowledge Base}

Corresponding with our main hypothesis, named a moderate earthquake, we consider only one seismic zone. This hypothesis leads to simplify the generic form of each critical component.

We propose a specific approach to enrich the database of component, especially when there is a lack of disaster data. In order to systemize the analyses on the behavior of a component, in case of earthquake, a guide based on its main characteristics (geometry, mass, materials, type of anchorage and functionality) allows to define the score of this generic component.

\subsection{The Method Itself}

Specific adaptations of the method are added to take into account:

- ageing of the component;

- cumulative effect of the PMF;

- importance of moderate earthquake, which is considered at the project level likewise the site affects;

- weight of the location of a component in the building, which depends on its height;

- capacity of defining generic model of a system, in order to accelerate the application.

\section{Conclusions}

The objective methods that treat the functional vulnerability of buildings are rare relatively to those that focus on the structural vulnerability. Even when a methodological support exists, another difficulty appears for the achievement of the method, which is the insufficiency of knowledge (shortcoming of data).
Beside, the available data are often come from a major earthquake more than a moderate one.

This method is dedicated to the technical staff of a hospital, giving them the ability to draw up a rough analysis of the vulnerability of the system. This short cut allows them to gain time and money, and then they will have recourse to specialist only for specific and critical analyses.

\section{References}

[1] Naumoski, N., Foo, S., and Saatcioglu, M. 2002. "Assessment and Mitigation of Earthquake Risk Related to Functional and Operational Buildings Components: A Canadian Perspective." Document written for the Office of the Protection of Critical Infrastructure and Civil Protection, University of Ottawa, Canada.

[2] WHO (World Health Organization). 2004. Guidelines for Seismic Vulnerability Assessment of Hospitals Kathmandu. Report of WHO and NSET (National Society for Earthquake Technology), Nepal.

[3] Faccioli, E., and Pessina, V. 1999. "The Catania Project: Seismic Scenarios for a High Risk in the Mediterranean Area." Presented at National Colloquium $\mathrm{N}^{\circ} 5$ : Earthquake Engineering and Dynamic Response of Structures, Cachan, France.

[4] Mebarki, A. 2003. "Seismic Risk: Hazards, Vulnerability and Help by GIS Maps." Presented at International Colloquium "Risk Vulnerability and Reliability in the Construction to a Disaster Reduction", Alger, Algeria.

[5] Whitman, R. V., Reed, J. W., and Hong S. T. 1973. "Earthquake Damage Probability Matrices." Presented at the 5th World Conference on Earthquake Engineering, Rome, Italy.

[6] Federal Emergency Management Agency. 1997. Earthquake Loss Estimation Methodology, Technical Manual. Washington, D.C.: National Institute of Building Sciences for the Federal Emergency Management Agency.

[7] GNDT (Gruppo Nazionale per la Difesadai Terremoti). 1993. Seismic Risk of Public Buildings-Part I: Methodological Aspect. Bologna: National Group for Difesa Dai Earthquakes, Center Services Quasco.

[8] Abdulhamid, R. A. 2011. "Contribution to the Assessment of the Seismic Vulnerability of a Hospital Building." Ph.D. thesis, University of Grenoble, France.

[9] Porter, K. A. 2003. Seismic Vulnerability Handbook Earthquake Engineering, edited by Chen, W. F., and Scawthorn, C. Boca Raton: CRC Press.

[10] Gayle S., and Johnson, S. G. 2003. Equipment and 
Systems Handbook Earthquake Engineering, edited by Chen, W. F., and Scawthorn, C. Boca Raton: CRC Press.

[11] Johnson, G. S., Sheppard, R. E., Quilici, M. D., Eder, S. J., and Scawthon, C. R. 1999. Seismic Reliability
Assessment of Critical Facilities: A Handbook, Supporting Documentation, and Model Code Provisions. Technical Report of MCEER 99-0008, University at Buffalo, USA. 